\title{
Influence of Isomorphic Atom Substitution on Lattice Anisotropy of Thallium Dichalcogenide Layered Mixed Crystals
}

\author{
N.M. GASAnLY* \\ Department of Physics, Middle East Technical University, 06531 Ankara, Turkey
}

(Received December 23, 2005; revised version July 13, 2006)

Compositional variation of the lattice parameters of $\mathrm{TlBX}_{2}$-type (B $=\mathrm{Ga}$ or $\mathrm{In}$ and $\mathrm{X}=\mathrm{S}$ or $\mathrm{Se}$ ) mixed crystals with monoclinic structure were studied by X-ray diffraction technique. The lattice anisotropy $(c / b)$ of these mixed crystals changes linearly with substitution of the atoms located both at the centers and the vertices of the corresponding $\mathrm{BX}_{4}$ tetrahedra. A brief survey of the important features of the effect of isomorphic atom substitution on the lattice anisotropy and the unit cell volume of $\mathrm{TlBX}_{2}$ type mixed crystals with layered structure was presented.

PACS numbers: $61.10 . \mathrm{Nz}, 61.66 . \mathrm{Dk}, 61.50 . \mathrm{Ks}$

\section{Introduction}

$\mathrm{TlBX}_{2}$-type compounds, where $\mathrm{B}=\mathrm{Ga}$ or $\mathrm{In}$ and $\mathrm{X}=\mathrm{S}$ or Se, crystallize in a monoclinic $\left(\mathrm{TlGaS}_{2}, \mathrm{TlGaSe}_{2}, \mathrm{TlInS}_{2}\right)$ or tetragonal $\left(\mathrm{TlInSe}_{2}\right)$ cell [1-3]. The lattice of the first three compounds consists of strictly periodic two-dimensional layers arranged parallel to the (001) plane. Each successive layer is rotated by $90^{\circ}$ with respect to the previous one. Interlayer bonding is formed between $\mathrm{Tl}$ and $\mathrm{B}$ atoms while the bonding between $\mathrm{B}$ and $\mathrm{X}$ atoms is an intralayer. The views of the crystal structure in the $a c$ - and $a b$-planes are presented in Figs. 1 and 2. The fundamental structural unit of a layer is the $\mathrm{B}_{4} \mathrm{X}_{10}$ polyhedron representing a combination of four elementary tetrahedra $\mathrm{BX}_{4}$ linked together by bridging $\mathrm{X}$ atoms. The $\mathrm{Tl}$ atoms are located in trigonal prismatic voids resulting from the combination of the $\mathrm{B}_{4} \mathrm{X}_{10}$ polyhedra into a layer. These atoms form nearly planar chains along the [110] and [110] directions.

*e-mail: nizami@metu.edu.tr; on leave from Physics Department, Baku State University, Baku, Azerbaijan 


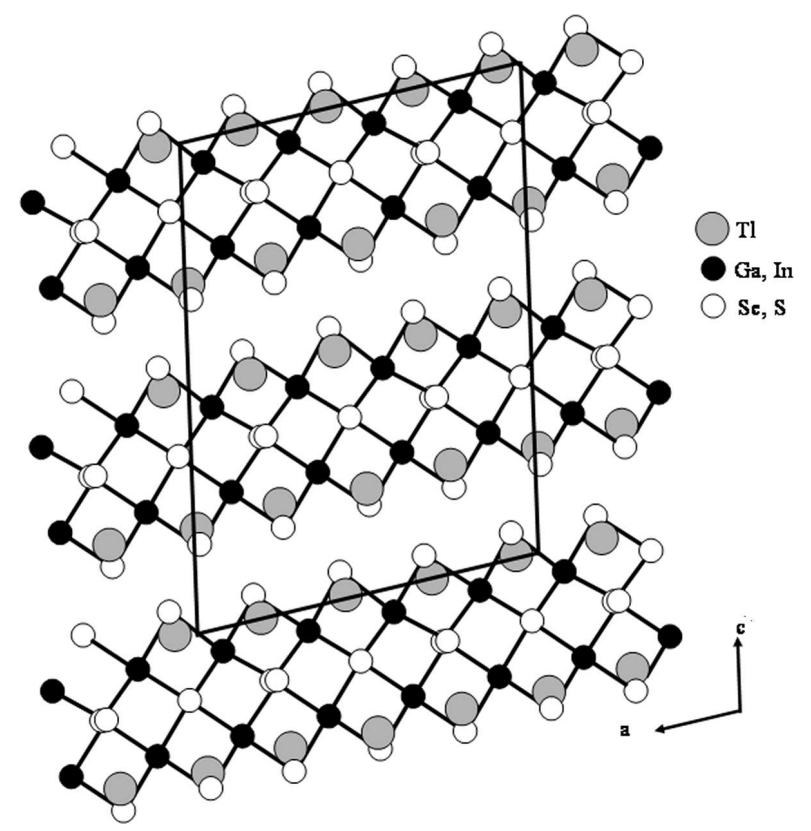

Fig. 1. Projection of the crystal structure of $\mathrm{TlGaSe}_{2}, \mathrm{TlGaS}_{2}$, and $\mathrm{TlInS} \mathrm{I}_{2}$ on ac-plane.

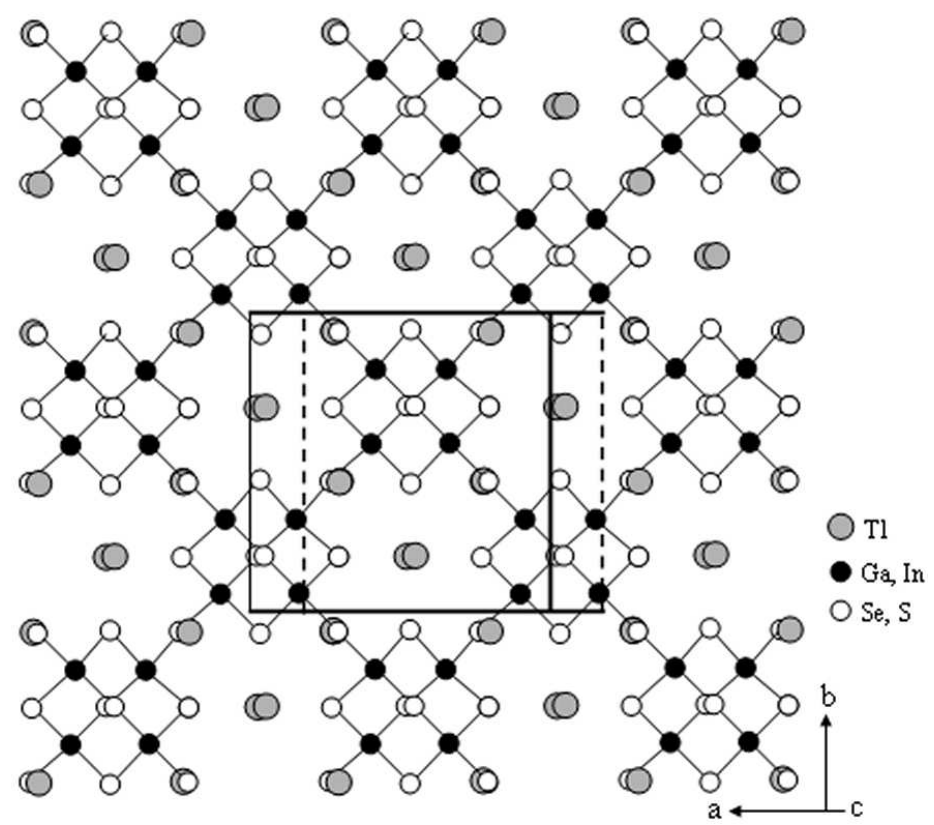

Fig. 2. Projection of the crystal structure of $\mathrm{TlGaSe}_{2}, \mathrm{TlGaS}_{2}$, and $\mathrm{TlInS} \mathrm{I}_{2}$ on $a b$-plane. 
The monoclinic $\mathrm{TlGaS}_{2}$ and $\mathrm{TlGaSe}_{2}, \mathrm{TlGaS}_{2}$ and $\mathrm{TlInS}_{2}$ form a series of $\mathrm{TlGa}\left(\mathrm{S}_{1-x} \mathrm{Se}_{x}\right)_{2}$ and $\mathrm{TlGa}_{1-x} \operatorname{In}_{x} \mathrm{~S}_{2}$ mixed crystals, respectively, with no restrictions on the concentration of the components: $0 \leq x \leq 1[4,5]$. The tetragonal TIInSe $e_{2}$ and monoclinic TIInS 2 compounds form a continuous series of mixed crystals $\operatorname{TIIn}\left(\mathrm{Se}_{1-x} \mathrm{~S}_{x}\right)_{2}(0 \leq x \leq 1)[6]$. The transformation from the tetragonal to monoclinic structure occurs at $x \approx 0.25$. The study of phase diagram in the $\operatorname{TlIn}_{1-x} \mathrm{Ga}_{x} \mathrm{Se}_{2}$ mixed crystals has shown that eutectic phase exists in the limited regions of $0-55.0$ and $70.0-100 \mathrm{~mol} \% \mathrm{TlInSe}_{2}$ [7]. In the first and second regions, $\mathrm{TlIn}_{1-x} \mathrm{Ga}_{x} \mathrm{Se}_{2}$ crystallize in the monoclinic and tetragonal structures, respectively.

The aim of the present paper was to study the influence of isomorphic atom substitution (gallium by indium and sulfur by selenium) on the lattice anisotropy and unit cell volume of $\operatorname{TlIn}_{1-x} \mathrm{Ga}_{x} \mathrm{Se}_{2}, \mathrm{TlGa}\left(\mathrm{S}_{1-x} \mathrm{Se}_{x}\right)_{2}, \mathrm{TlGa}_{1-x} \mathrm{In}_{x} \mathrm{~S}_{2}$, and $\operatorname{Tl} I n\left(\mathrm{Se}_{1-x} \mathrm{~S}_{x}\right)_{2}$ layered mixed crystals.

\section{Experimental details}

$\operatorname{TlIn}_{1-x} \mathrm{Ga}_{x} \mathrm{Se}_{2}(0.5 \leq x \leq 1), \operatorname{TlGa}\left(\mathrm{S}_{1-x} \mathrm{Se}_{x}\right)_{2}(0 \leq x \leq 1)$, $\mathrm{TlGa}_{1-x} \operatorname{In}_{x} \mathrm{~S}_{2}(0 \leq x \leq 1)$, and $\operatorname{TIIn}\left(\mathrm{Se}_{1-x} \mathrm{~S}_{x}\right)_{2} \quad(0.3 \leq x \leq 1)$ polycrystals were obtained by direct fusion in evacuated capsules of particular high-purity elements (at least $99.999 \%$ ) taken in stoichiometric proportions. Single crystals were grown by a modified Bridgman method from the polycrystals. The resulting ingots appear from dark red to yellow in color, and the freshly cleaved surfaces are mirror-like. In the X-ray powder diffraction experiment, a Philips PW1740 diffractometer with a monochromatized $\mathrm{Cu} K_{\alpha}$ radiation $(\lambda=0.15418 \mathrm{~nm})$ was used at a scanning speed of $0.02^{\circ} 2 \Theta / \mathrm{s}$. We have recorded X-ray powder diffractograms in the $2 \Theta$ range from 10 to $75^{\circ}$ of five $\mathrm{TlGa}_{1-x} \mathrm{In}_{x} \mathrm{Se}_{2}$, nine $\mathrm{TlGa}\left(\mathrm{S}_{1-x} \mathrm{Se}_{x}\right)_{2}$, eleven $\mathrm{TlGa}_{1-x} \mathrm{In}_{x} \mathrm{~S}_{2}$, and seven $\operatorname{TIIn}\left(\mathrm{Se}_{1-x} \mathrm{~S}_{x}\right)_{2}$ samples of mixed crystals having different compositions. The lattice parameters of the monoclinic unit cell were calculated by using a least squares computer program "Treor 90". The uncertainties in the calculated lattice parameters were in the range from 0.0003 to $0.012 \mathrm{~nm}$.

\section{Results and discussion}

The variations of the lattice parameters $(a, b$, and $c)$ and monoclinic angle $\beta$ of $\operatorname{TlIn}_{1-x} \mathrm{Ga}_{x} \mathrm{Se}_{2}(A), \operatorname{TlGa}\left(\mathrm{S}_{1-x} \mathrm{Se}_{x}\right)_{2}(B), \mathrm{TlGa}_{1-x} \mathrm{In}_{x} \mathrm{~S}_{2}(C)$, and $\operatorname{Tl} I n\left(\mathrm{Se}_{1-x} \mathrm{~S}_{x}\right)_{2}(D)$ mixed crystals with monoclinic structure as a function of their compositions are plotted in Figs. 3 and 4. Presently evaluated lattice parameters $a=1.0312, b=1.0431, c=1.5072 \mathrm{~nm}$ and $\beta=99.61^{\circ}\left(\mathrm{TlGaS}_{2}\right)$, $a=1.0756, b=1.0730, c=1.5596 \mathrm{~nm}$ and $\beta=99.92^{\circ}\left(\mathrm{TlGaSe}_{2}\right)$, and $a=1.0942, b=1.0484, c=1.5606 \mathrm{~nm}$ and $\beta=100.70^{\circ}\left(\mathrm{TlInS}_{2}\right)$ agree well with the corresponding data of Refs. $[1,2]$. The main difference between mixed crystals $A$ and $C$ and mixed crystals $B$ and $D$ is the fact that in the mixed crystals $A$ and $C$ the substitution of atoms (gallium by indium) located at the centers of 


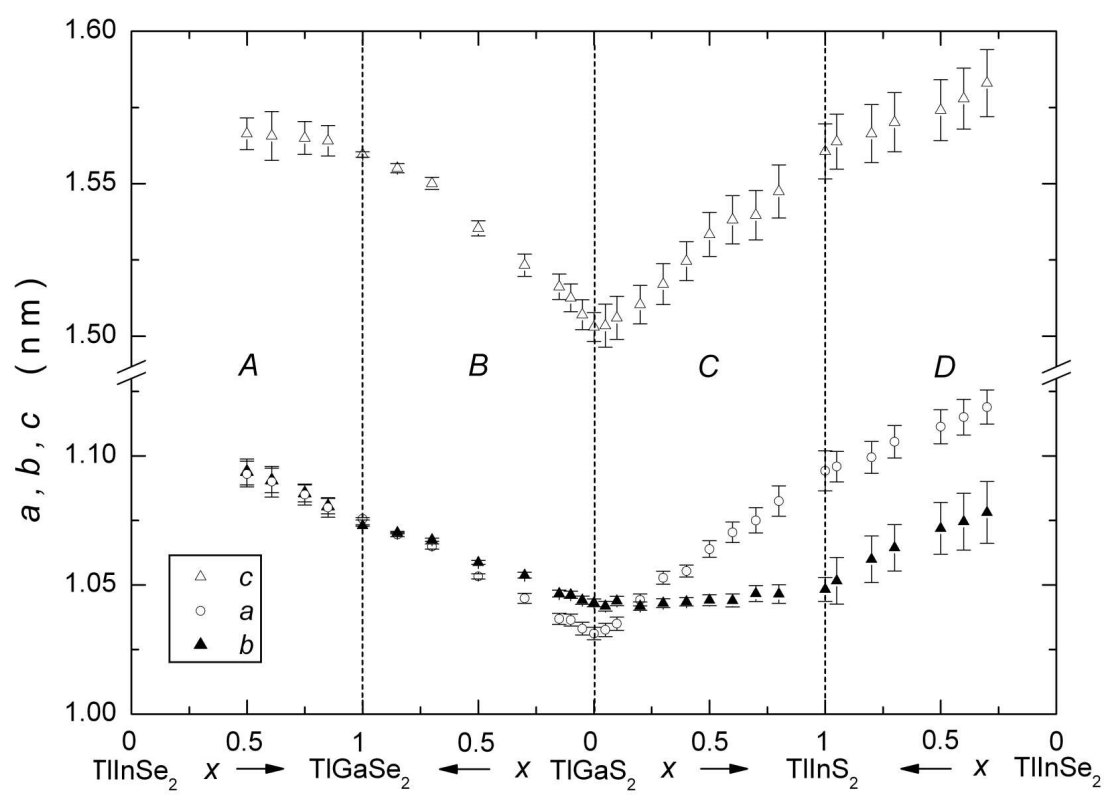

Fig. 3. Compositional variations of the lattice parameters $(a, b$, and $c)$ of $\operatorname{TlIn}_{1-x} \mathrm{Ga}_{x} \mathrm{Se}_{2}(A), \operatorname{TlGa}\left(\mathrm{S}_{1-x} \mathrm{Se}_{x}\right)_{2}(B), \mathrm{TlGa}_{1-x} \operatorname{In}_{x} \mathrm{~S}_{2}(C)$, and $\operatorname{TlIn}\left(\mathrm{Se}_{1-x} \mathrm{~S}_{x}\right)_{2}(D)$ mixed crystals.

$\mathrm{GaSe}_{4}, \mathrm{InSe}_{4}$ and $\mathrm{GaS}_{4}, \mathrm{InS}_{4}$ tetrahedra, respectively, takes place; whereas in the mixed crystals $B$ and $D$ the substitution of atoms (sulfur by selenium) located in the vertices of the $\mathrm{GaS}_{4}, \mathrm{GaSe}_{4}$ and $\mathrm{InS}_{4}, \mathrm{InSe}_{4}$ tetrahedra, respectively, takes place. As seen from Figs. 3 and 4 , the lattice parameters $a, b, c$ and monoclinic angle $\beta$ of $A$ and $C$, and $B$ and $D$ mixed crystals increase at the substitution of gallium atoms by indium and of sulfur atoms by selenium, respectively. These observations may be accounted for considering the covalent radius of replacing atoms, which are equal to $0.126,0.144,0.104$, and $0.114 \mathrm{~nm}$ for gallium, indium, sulfur, and selenium, respectively. The larger atoms replacing the smaller ones at the centers of the $\mathrm{BX}_{4}$ tetrahedra (gallium by indium in $A$ and $C$ mixed crystals) and at the vertices of the $\mathrm{BX}_{4}$ tetrahedra (sulfur by selenium in $B$ and $D$ mixed crystals) cause the observed lattice expansion in the mixed crystals studied.

The variations of the unit cell volume $(V)$ and lattice anisotropy $(c / b)$ of $A, B, C$, and $D$ mixed crystals as a function of their compositions are presented in Fig. 4. As seen from this figure, the unit cell volume of $A$ and $C$, and $B$ and $D$ mixed crystals also increase at the substitution of gallium atoms by indium and of sulfur atoms by selenium, respectively. As for the variations of the lattice anisotropy, these dependencies on composition of mixed crystals show quite different behavior. The lattice anisotropy for $A$ and $C$ mixed crystals noticeably decreases or increases at the substitution of gallium atoms by indium ones, re- 


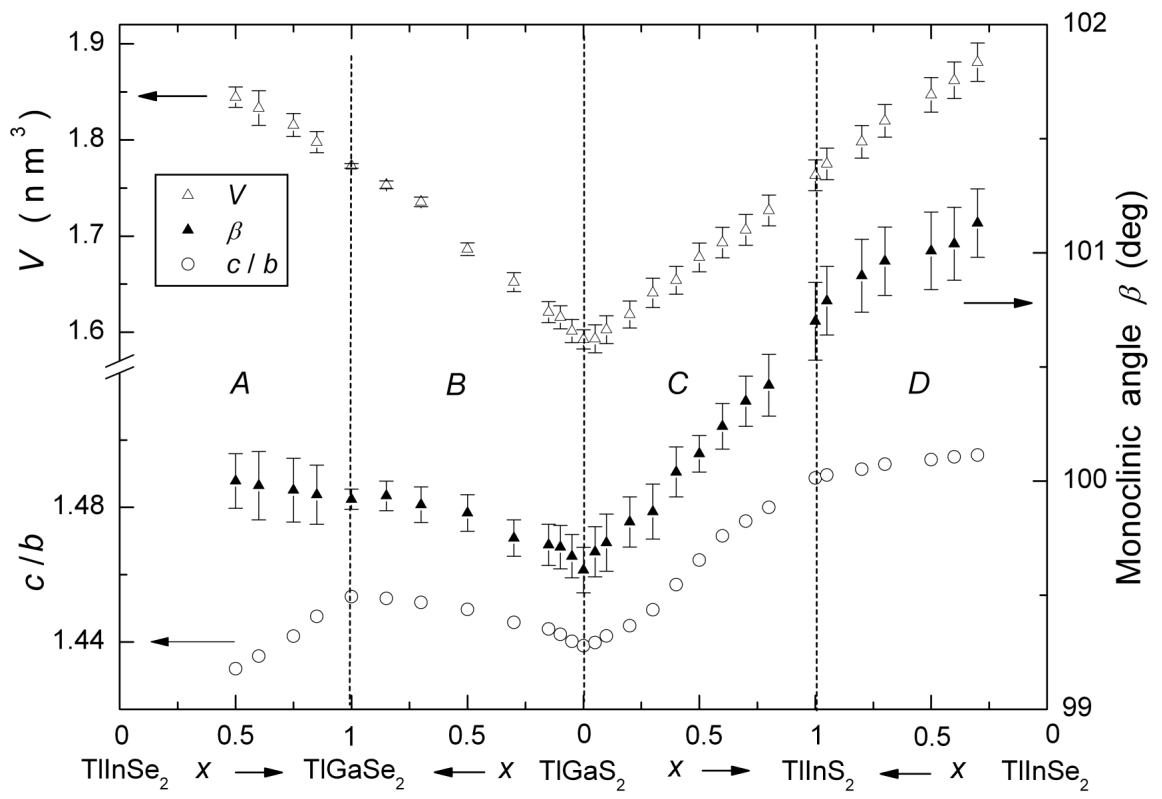

Fig. 4. Compositional variations of the unit cell volume $(V)$, monoclinic angle $(\beta)$ and lattice anisotropy $(c / b)$ of $\operatorname{TlIn}_{1-x} \mathrm{Ga}_{x} \mathrm{Se}_{2}(A), \operatorname{TlGa}\left(\mathrm{S}_{1-x} \mathrm{Se}_{x}\right)_{2}(B), \mathrm{TlGa}_{1-x} \operatorname{In}_{x} \mathrm{~S}_{2}(C)$, and $\operatorname{TIIn}\left(\mathrm{Se}_{1-x} \mathrm{~S}_{x}\right)_{2}(D)$ mixed crystals.

spectively. As for $B$ and $D$ mixed crystals, in both of them the lattice anisotropy shows slight increase at the substitution of sulfur atoms by selenium ones. Thus, when the atoms at the centers of $\mathrm{Ga}(\mathrm{In}) \mathrm{Se}_{4}$ and $\mathrm{Ga}(\mathrm{In}) \mathrm{S}_{4}$ tetrahedra are replaced by the larger atoms (gallium by indium) in $A$ and $C$ mixed crystals, the unit cell becomes less or more anisotropic, respectively, depending on the types of atoms (selenium or sulfur) located at the vertices of corresponding tetrahedra. On the other hand, when the atoms at the vertices of $\mathrm{Ga}\left(\mathrm{S}_{4}\right) \mathrm{Se}_{4}$ and $\operatorname{In}\left(\mathrm{S}_{4}\right) \mathrm{Se}_{4}$ tetrahedra are replaced by the larger atoms (sulfur by selenium) in $B$ and $D$ mixed crystals, the unit cell becomes somewhat more anisotropic, which does not depend on the types of atoms (gallium or indium) located at the centers of corresponding tetrahedra.

As we mentioned above, in the crystals studied interlayer bonding is formed between $\mathrm{Tl}$ and $\mathrm{S}(\mathrm{Se})$ atoms whereas the bonding between $\mathrm{Ga}(\mathrm{In})$ and $\mathrm{S}(\mathrm{Se})$ atoms is responsible for intralayer interaction (Figs. 1 and 2). In the case of $A$ mixed crystals (Tl-Se interlayer bonding) replacement of Ga atoms by In atoms leads to the noticeable increase in lattice parameter $b$ while for the parameter $c$ this increase is less pronounced (Fig. 3). Therefore, the lattice anisotropy $c / b$ decreases for $A$ mixed crystals comprising selenium atoms (Fig. 4). On the other hand, for $C$ mixed crystals (Tl-S interlayer bonding) replacement of Ga atoms by In atoms results in the slight increase in lattice parameter $b$ while for the parameter $c$ this 
increase is more pronounced (Fig. 3). Hence the lattice anisotropy $c / b$ increases for $C$ mixed crystals comprising sulfur atoms (Fig. 4). These observations imply that the different behavior of the lattice anisotropy $c / b$ with respect to the composition of mixed crystals is related to the chalcogenide atoms (selenium or sulfur) taking part in interlayer and intralayer bondings.

It is worthy noting that the rate of change in the lattice anisotropy $c / b$ with composition for $B$ and $C$ mixed crystals, having monoclinic structure throughout the whole composition range, appears to be proportional to the covalent radii difference, $\Delta R$, of atoms substituted $\left(\Delta R=R_{\mathrm{Se}}-R_{\mathrm{S}}=0.010 \mathrm{~nm}\right.$ and $\Delta R=$ $R_{\mathrm{In}}-R_{\mathrm{Ga}}=0.018 \mathrm{~nm}$ for $B$ and $C$ mixed crystals, respectively). The same tendency is also observed for $A$ and $D$ mixed crystals in the studied composition ranges of $0.5 \leq x \leq 1$ and $0.3 \leq x \leq 1$, respectively (Fig. 4).

Now let us consider the variation of the lattice volume on the compositions of the mixed crystals studied. It is well known from the literature that $\mathrm{TlGaSe}_{2}$ and $\mathrm{TlInS}_{2}$ sequentially undergo low-temperature transitions to an incommensurate phase and a ferroelectric phase at $T_{\mathrm{i}}=115 \mathrm{~K}, T_{\mathrm{c}}=108 \mathrm{~K}$ and $T_{\mathrm{i}}=213 \mathrm{~K}$, $T_{\mathrm{c}}=189 \mathrm{~K}$, respectively [8-11]. However, in $\mathrm{TlGaS}_{2}$ crystals no evidence for a soft mode behavior has been found. Yee and Albright [12] have studied the bonding and structure of $\mathrm{TlGaSe}_{2}$ crystal by tight-binding calculations and have revealed that the ferroelectric phase transition is essentially associated with the slippage of the two thallium atom channels along the [110] and [11̄0] directions, respectively (see Figs. 1 and 2). Since in $\mathrm{TlGa}\left(\mathrm{S}_{1-x} \mathrm{Se}_{x}\right)_{2}$ and $\mathrm{TlGa}_{1-x} \mathrm{In}_{x} \mathrm{~S}_{2}$ mixed crystals the unit cells get smaller with increase in sulfur and gallium contents, respectively, the average distances between the thallium atom channels also get shorter as compared with those of $\mathrm{TlGaSe}_{2}$ and $\mathrm{TlInS}_{2}$ crystals. Therefore, the thallium atom channel slippages in the smaller unit cell do not easily take place so that they may not be sufficient for destroying the inversion center, which is necessary for the occurrence of ferroelectricity. This assumption is in agreement with the absence of ferroelectric phase transitions with soft mode features in $\mathrm{TlGaS}_{2}$ crystal [13] and $\mathrm{TlGa}\left(\mathrm{S}_{1-x} \mathrm{Se}_{x}\right)_{2}$ mixed crystals with compositions $x<0.75$ [14].

\section{Acknowledgments}

The author is grateful to Prof. A. Culfaz and Prof. H. Ozkan for valuable discussions.

\section{References}

[1] D. Muller, F.E. Poltmann, H. Hahn, Z. Naturforsch. 29b, 117 (1974).

[2] D. Muller, H. Hahn, Z. anorg. Allg. Chemie 438, 258 (1978).

[3] D. Muller, G. Eulenberger, H. Hahn, Z. anorg. Allg. Chemie 398, 207 (1973).

[4] N.M. Gasanly, A. Culfaz, H. Ozkan, S. Ellialtioglu, Cryst. Res. Technol. 29, K51 (1994).

[5] N.M. Gasanly, N.S. Yuksek, Acta Phys. Pol. A 108, 997 (2005). 
[6] E. Kerimova, S. Mustafaeva, D. Guseinova, S. Babaev, T. Mamedov, Z. Salaeva, K. Allakhverdiev, Phys. Status Solidi A 179, 199 (2000).

[7] B. Abay, H. Guder, H. Efeoglu, Y.K. Yogurtcu, J. Phys. Chem. Solids 62, 747 (2001).

[8] V.M. Burlakov, N.M. Gasanly, M.P. Yakheev, Sov. Phys.-Solid State 32, 29 (1990).

[9] V.M. Burlakov, A.P. Ryabov, M.P. Yakheev, E.A. Vinogradov, N.N. Melnik, Phys. Status Solidi B 153, 27 (1989).

[10] F.A. Mikailov, E. Basaran, E. Senturk, J. Phys., Condens. Matter 13, 727 (2001).

[11] F.A. Mikailov, E. Basaran, E. Senturk, L. Tumbek, T. Mammadov, V. Aliev, Phase Transit. 76, 1057 (2003).

[12] K.A. Yee, A. Albright, J. Am. Chem. Soc. 113, 6474 (1991).

[13] A. Aydinli, R. Ellialtioglu, K.R. Allakhverdiev, S. Ellialtioglu, N.M. Gasanly, Solid State Commun. 88, 387 (1993).

[14] A.A. Volkov, Yu.G. Goncharov, G.V. Kozlov, K.R. Allakhverdiev, R.M. Sardarly, Sov. Phys.-Solid State 26, 1668 (1984). 